\title{
Intelligent Healthcare Service by using Collaborations between IoT Personal Health Devices
}

\author{
Byung Mun Lee* and Jinsong Ouyang \\ Dept. of Computer Science, Gachon University, Korea, \\ Dept. of Computer Science, California State University Sacramento, USA \\ *Corresponding Author:bmlee@gachon.ac.kr,jouyang@@csus.edu
}

\begin{abstract}
Management of chronic diseases is important to self-management for health. The IoT concept plays a significant role in self-management for health. In order to accomplish it, personal health devices need two functions such as application network protocol and intelligent service. But, most of them have only simple function such as indicating measured data and storing data temporarily. In this research, we proposed an intelligent service model for healthcare which gives an effective feedback to an individual. In order to do this, we introduced the collaboration protocol which transfers risk factors between IoT personal health devices. In addition to this, we proposed intellectualized service application algorithm which will be operated in the personal health device. Finally, based on the findings of the experiment, the effectiveness was confirmed on proposed model.
\end{abstract}

Keywords: Ubiquitous Health, Chronic Disease, Collaboration Protocol, IoT, Intelligence

\section{Introduction}

Ubiquitous health (UH) service was a model in which individual medical data was measured by a ubiquitous personal health device (UHD), and then sent to the health server to provide feedback to medical experts and patients [1]. Thus, most researches were focused on the function of sending the measured biomedical data to the server [1, $2,3]$. Due to this reason, the analysis and processing function of medical data were mostly conducted in the server.

As the concept of IoT (Internet of Things) was recently introduced, researches which attempt to apply the IOT model in different fields are being progressed $[4,5,6]$. If IoT technique is applied to UH, then UHD will break away from the simple functions of indicating measured data and sending them to the server and execute autonomous information exchange with neighboring systems (UHDs, gateway, server) and provide comprehensively assessed feedback immediately to the patient [4]. For instance, a blood pressure which is above $140 \mathrm{mmHg}$ is generally assessed as hypertension [7]. If the blood pressure measured from a patient with symptoms of diabetes mellitus is $135 \mathrm{mmHg}$, then an intellectualized feedback service which assesses the condition as stage 1 hypertension instead of prehypertension can be provided [7]. This can be provided only when the mutual relationship between risk factors of the disease is identified.

In this research, we propose an intelligent healthcare service model that can enable personal health device to recognize the relationship between mutual diseases and risk factors and provide intellectualized feedback to the patient. In addition, suggestion is 
made for the assessment algorithm of intellectualized processing essential during the modeling procedure and collaboration application protocol between personal health devices. Furthermore, it is presented the effectiveness of the proposed model through experiments

In related researches presented in Chapter 2, the characteristics and diagnostic criteria of metabolic syndrome, a major risk factor of chronic diseases, are explored. Moreover, structural characteristics will be observed to identify the degree of suitability of conceptual model of IOT on U-Health. In Chapter 3, suggestion will be made to intellectualized collaboration protocol that can be operated in IOT based UHD. In Chapter 4, virtual data is utilized to conduct experiment and experiment results are analyzed. Lastly, the conclusion will be presented in Chapter 5.

\section{Related Researches}

The metabolic syndrome refers to the condition where more than 3 amongst the 5 factors (glucose, blood pressure, obesity, triglycerides, and HDL cholesterol) are above standard values $[4,7]$. A metabolic syndrome can cause various complications such as cardiovascular disease and brain stroke [8]. Therefore, it is important to manage these risk factors during daily life. However, since the 3 risk factors hold close mutual relationship, it is necessary to give complex consideration to various risk factors when setting the diagnostic criteria. Additionally, in order to look into whether processing about the complex diagnostic criteria can be applied to the IOT machine, structural characteristics of IOT will be explored.

\subsection{Risk factors of Metabolic Syndrome}

Generally, states of diseases are assessed according to the degree of error of the measurement value in comparison to the normal range [4]. When blood pressure is taken as an example, it is assessed as stage 1 hypertension when the measured systolic blood pressure (SBP) is over $140 \mathrm{mmHg}$ and the diastolic blood pressure (DBP) is over than $90 \mathrm{mmHg}[4,7]$. However, in case of patients diagnosed with diabetes mellitus or chronic kidney disease, it should be assessed as stage 1 hypertension when they are over $130 \mathrm{mmHg}$ or $80 \mathrm{mmHg}$, so that accurate assessment takes places when the fixed range is readjusted $[4,7]$.

This displays how conducting comprehensive assessment on various risk factors instead of only the measured values during the stage of diagnosing the degree of disease will provide accurate information to the patient. Due to these reasons, the assessment procedure for hypertension gives comprehensive consideration to SBP, DBP, prevalence rate following the age and gender, the current state of personal disease (diagnostic results for obesity and diabetes mellitus), and family history as Figure 1 [7]. But most medical sensor devices do not give comprehensive assessment.

Even in case of type 2 diabetes which consists for more than $90 \%$ of diabetes disease, consideration is made for glucose measurement value along with risk factors such as obesity, drinking, age, gender, personal disease, and family history $[9,10]$. Table 1 is a display of each evaluative result which is in accordance to the range of measurement values for blood pressure, glucose, BMI(Body Mass Index), and waise circumference. In terms of the assessment scale for obesity, BMI and waist circumference are applied. As a result, obesity was classifed as a risk factor which caused influence in diabetes and blood pressure $[10,11]$. 


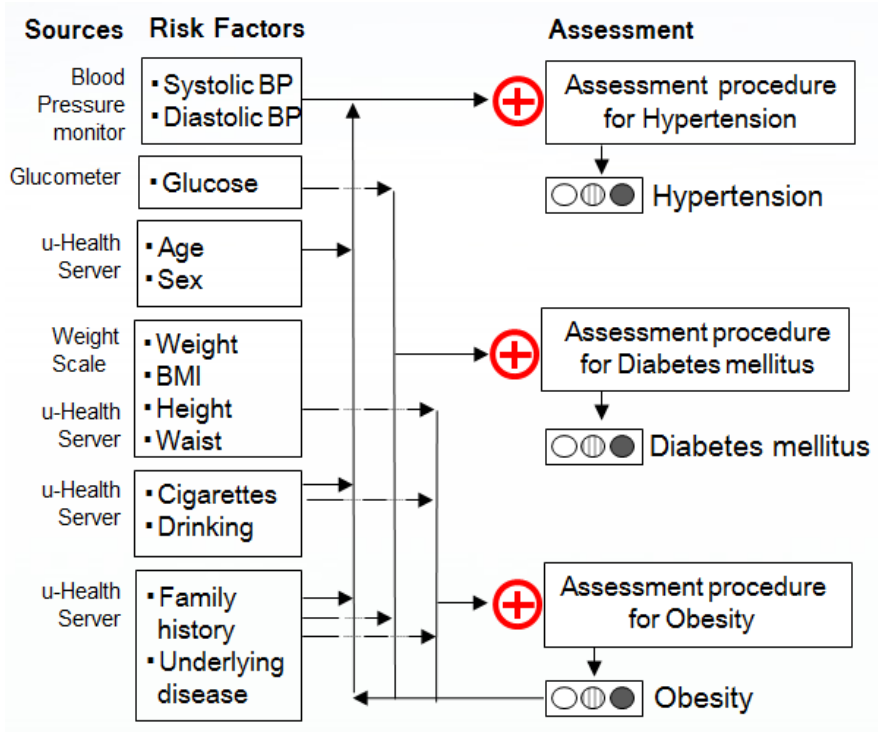

(1D) Risk Level (Normal, Pre stage, Stage 1 or 2 or 3 )

Figure 1. Risk factors of Hypertension, Diabetes and Obesity

\section{Table 1. Classifications of Blood Pressure, Diabetes and Obesity}

\begin{tabular}{|c|c|c|c|c|c|c|}
\hline \multicolumn{2}{|c|}{ Risk Factors } & Normal & Pre stage & Stage 1 & Stage 2 & Stage 3 \\
\hline \multirow{2}{*}{$\begin{array}{c}\text { Blood } \\
\text { Pressure }\end{array}$} & SBP & $<120$ & $120-139$ & $140-159$ & $\geq 160$ & \\
\cline { 2 - 7 } Glucose & DBP & $<80$ & $80-89$ & $90-99$ & $\geq 100$ & \\
\cline { 2 - 7 } & FPG & $<100$ & $100-125$ & $\geq 126$ & & \\
\hline \multicolumn{2}{|c|}{ BMI } & $18.5-24.9$ & $25.0-29.9$ & $30.0-34.9$ & $35.0-39.9$ & $\geq 40$ \\
\hline \multicolumn{5}{|c|}{$\begin{array}{c}\text { Waist } \\
\text { Circumference }\end{array}$} & $\leq 40, \leq 35$ & \multicolumn{5}{|c|}{$>40,>35$} \\
\hline
\end{tabular}

FPG Fasting Plasma Glucose, 2h-PG 2 hours Plasma Glucose, BMI Body Mass Index

\subsection{Structural Characteristic of IoT}

IOT is a conceptual model which provides service for human beings through things, which possess innate roles, maintain mutually autonomous collaboration system centered on the network and minimize human intervention $[4,5]$. IOT can be applied to different types of services, and common application service elements such as network access management, power management, identification management, and security management must be acquired $[4,12]$. Moreover, there are also specific application service elements of the thing. For instance, in case of IOT sensor thing, sensor management and intelligent application of sending or handling measurement data are specific application service elements. Therefore, Figure 2 is a representation of classification between common application service elements and specific application the service elements.

WSN (Wireless Sensor Network) and M2M (Machine-to-Machine Communication) can be considered as other examples of IOT. In case of WSN, data measured from the sensor is sent to the server or the information provided by the server hold one-sided directivity as it is sent to the actuator. On the other hand, M2M holds bilateralness as processing is executed through autonomous communication between each device [5]. 
Therefore, the current u-Health model where data measured from the medical sensor is sent to the u-Health server can be considered approximate to WSN [5].

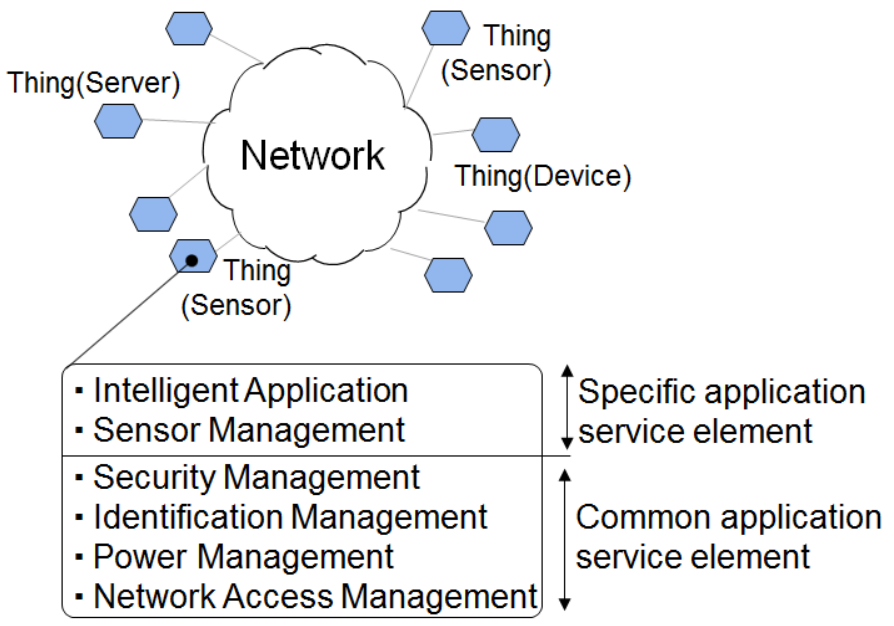

Figure 2. Conceptual Model for Internet of Things and its functionalities

However, the service elements of device and network have recently become superior and user demands became diversified and these conditions brought the need of more intellectualized and distributed processing [4]. This is suggesting a new direction for the u-Health service to change from the current WSN type to the M2m type in the future. Within this context, this study will suggest a new smart healthcare service model based on IOT

\section{Intelligent Healthcare Service}

\subsection{Collaboration model}

The Smart healthcare service model is a collaboration model which acquires risk factors needed for assessment from the network and reflects on them by real-time. Two types of factors are needed for this service. They are the collaboration protocol nee ded to acquire the risk factors and intelligent application needed for reflected intellectualized assessment. Figure 3 defined the IOT based service model regarding the risk factors of 3 major metabolic syndromes. First of all, IOT $_{\mathrm{XX}}$ is represented as a device which enables connection to the IOT network and autonomous collaboration and the functions of each device was indicted as xx. In Figure 3, (a) is a specific representation of this. In terms of the blood pressure monitor ( $\mathrm{IOT}_{\mathrm{BP}}$ ), only the measured blood pressure is not displayed. Instead, data on diabetes measured by the glucose meter $\left(\mathrm{IOT}_{\mathrm{GL}}\right)$ and the data on obesity measured by the weight scale (IOT $\left.\mathrm{OB}_{\mathrm{B}}\right)$ are requested to acquire the result [4]. If data cannot be immediately sent by other IOTs, information stored by gateway $\left(\mathrm{IOT}_{\mathrm{GW}}\right)$ is requested for and attained.=In addition to this, risk factors of the patient such as the gender, age, and family history are received by the u-Health server $\left(\mathrm{IOT}_{\mathrm{SVR}}\right)$. However, even when the IOT device is not within the network reception range or is turned off just as it is shown in (b) or (c), information sent from $\mathrm{IOT}_{\mathrm{GW}}$ or $\mathrm{IOT}_{\mathrm{SVR}}$ are applied to assess the measurement value 

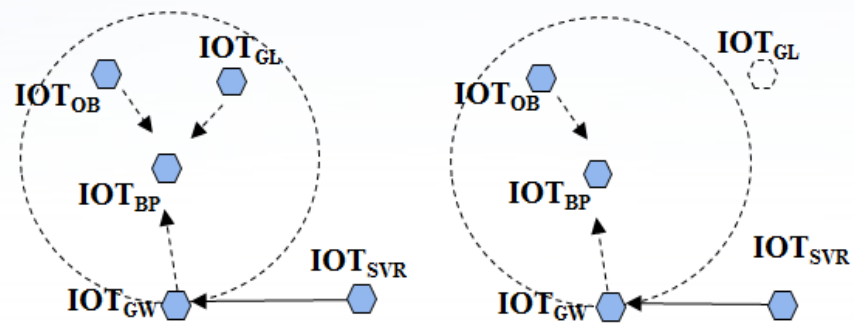

(a) All wireless IOT devices are involved

(b) Some wireless IOT devices are involved

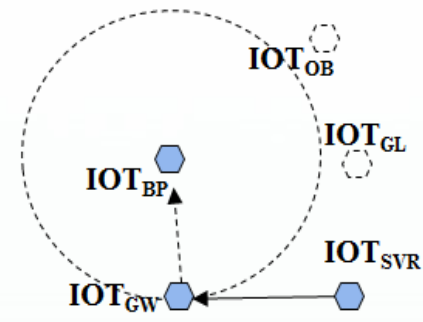

(c) Only wireless one is involved

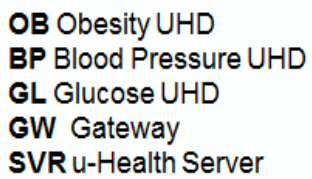

Figure 3. Collaboration model between IOTs for the smart healthcare service

IOT $_{\mathrm{XX}}$ must collect essential information by real-time to effectively construct a collaboration model. In order to do so, seamless collaboration protocol is needed between each $\mathrm{IOT}_{\mathrm{XX}}$ device. We propose the collaboration protocol at the next clause.

\subsection{Collaboration protocol between IoT personal health devices}

The collaboration protocol is an application protocol which sends and receives risk factor information needed between IOT devices [4]. Since u-Health devices used in home connect to the home networks like Bluetooth or Zigbee, the protocol suggested in this paper is an application protocol over the WSN. In Figure 4, (a) displays an example of a protocol process in the case of (b) of Figure 3. Once IOT $_{\mathrm{BP}}$ operates, Join message is broadcasted and make known to all devices in the network. During this time, IOT $_{\mathrm{OB}}$ and $\mathrm{IOT}_{\mathrm{GW}}$ receive the Join message, but $\mathrm{IOT}_{\mathrm{GL}}$ is not able to receive it. $\mathrm{IOT}_{\mathrm{BP}}$ measures the blood pressure and sends the Data.Req message so that risk factors needed for assessment are gathered.

$\mathrm{IOT}_{\mathrm{Oв}}$ and $\mathrm{IOT}_{\mathrm{GW}}$, which receive Data.Req message, send the Data.Ack message and the risk factor information of relevant patients stored by each device $=$ If risk factors about patients are not available, Data.Nak is sent to notify that there is no information available. In order to receive the newest information, Data.Req is sent each time the blood pressure was measured so that the latest information is reflected on the assessment. It was structured so that the Leave message is sent to make known that the network was left from when the power of the IOT $_{\mathrm{BP}}$ became switched off or measurement was terminated. It was designed so that the overall procedures can take place seamlessly and user convenience was optimized 


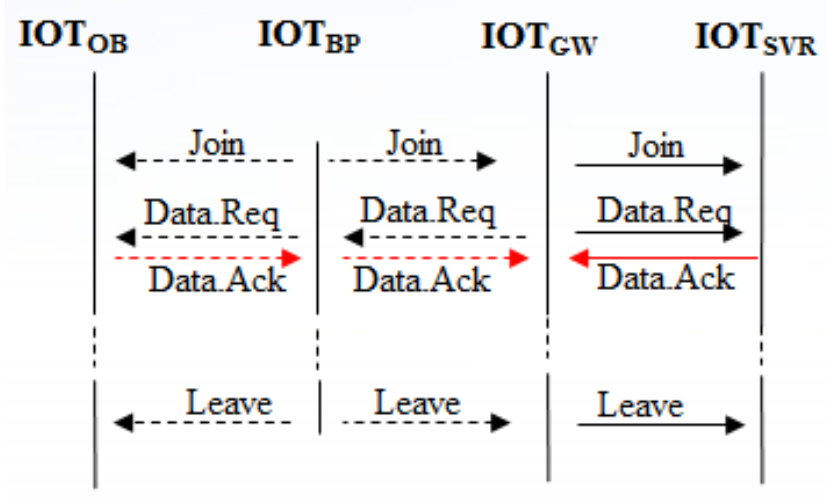

(a) Protocol between IOT devices

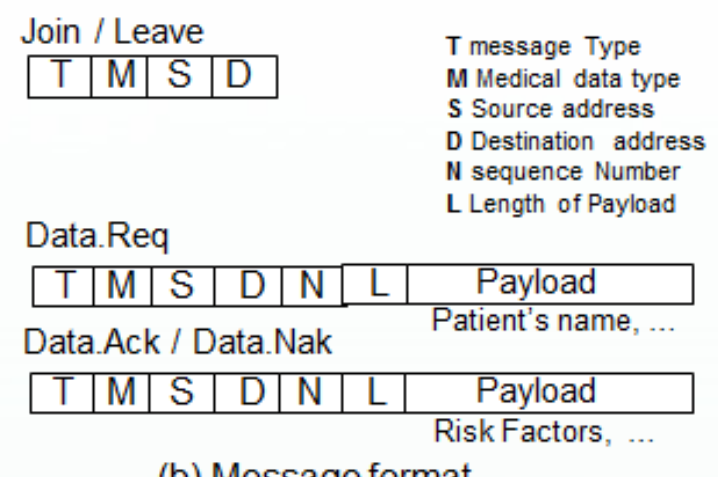

(b) Message format

Figure 4. Collaboration model between PHDs for the smart healthcare service

In Figure 4, (b) represents the format of the messages used during the transmission process of (a). The number of message type is 5 in this protocol. 'Join' and 'Leave' were defined without 'Payload' in order to minimize the transmission overhead. In medical data type (M), classification was made between blood pressure, glucose, and obesity. The source address (S) and destination address (D) of the IOT device were defined in the message. Additionally, the message includes the sequence number $(\mathrm{N})$ of each message, length of payload. And also, patient identification information and risk factors were defined in the Payload. Each header field was constructed as 1 byte and payload was defined as variable size. The information collected in form of this protocol is applied to provide comprehensive consideration to the relationship between mutual diseases and risk factors. In the next clause, definition of intelligent application which can manage this process will be explored

\subsection{Intellectualized service application}

Event-driven model is appropriate for the intelligent application operating in IOT since it has to take measurements by the sensor while collecting data by the use of network. Therefore, as it is displayed in Figure 5, the application is constructed as 5 major events and their event handlers for processing. The first event is a boot event which occurs when the power is turned on and it initializes the IOT device and broadcasts the Join message. In the measurement event which occurs when 
measurement button is pressed to measure the medical data, Data.Req message is sent and measurement function is activated.

DataAcquisition event occurs when the measurement becomes terminated and the measurement data is acquired from the sensor. Furthermore, Receive event also occurs when Data.Ack or Data.Nak sent from other device becomes received. Accordingly, Receive event must make progressing according to the types of received message. Join and Leave messages cause for IOT Member list available from the network to become added or deleted. Additionally, if the type of the received message is Data.Req, it is the request to send risk factors (measurement value or assessment result) of the patient designated in the Payload.

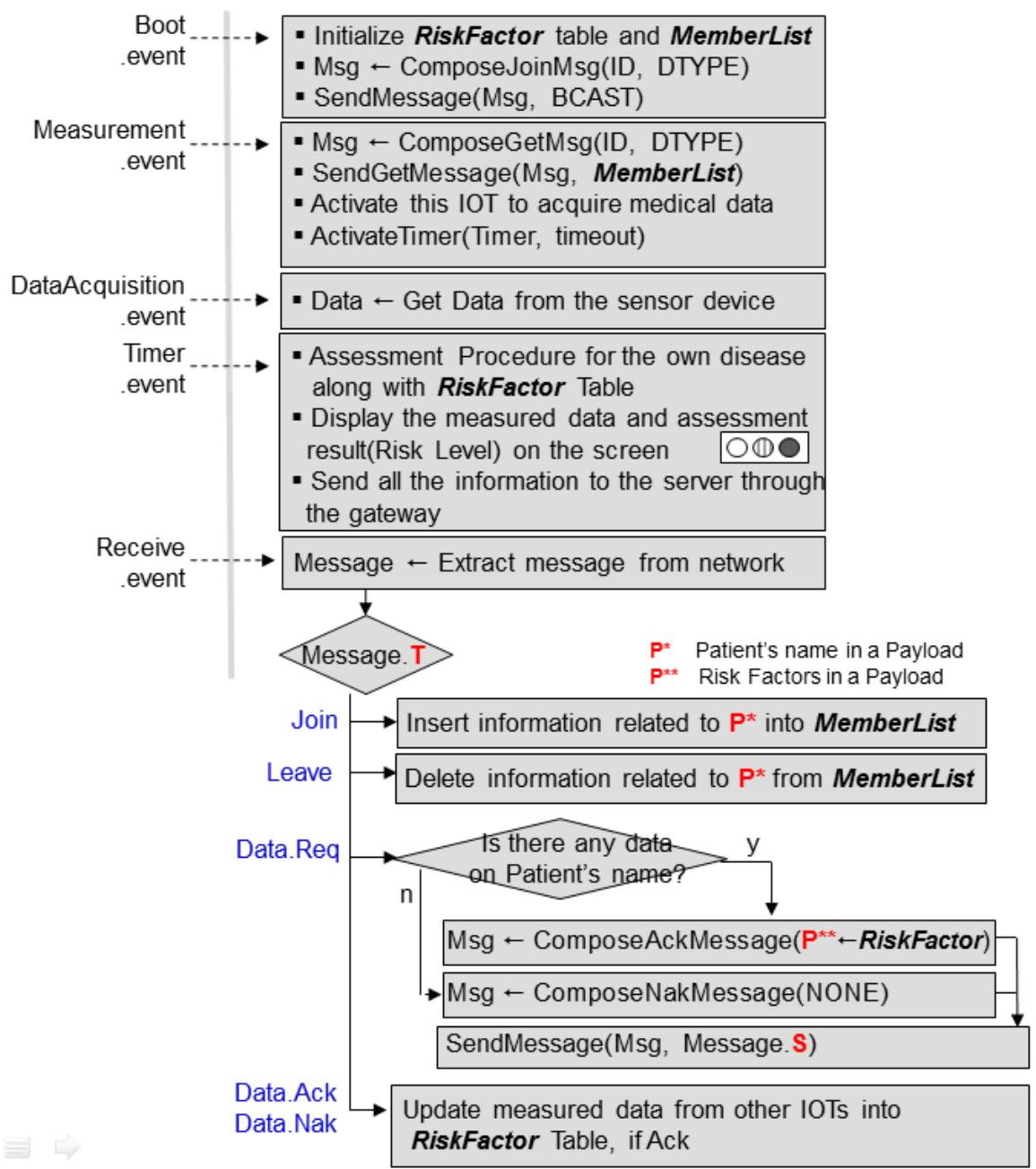

Figure 5. Process flow in an IOT sensor application

Therefore, risk factor table is searched and Data.Ack (or Data.Nak) message is composed and sent. Moreover, this algorithm was structured so that it does not suggest measurement value immediately upon the occurrence of DataAcquisition event and pass through assessment process after fixed timeout. In order to receive all the messages from other IOT devices in the network, time delay is needed. 


\section{Evaluations}

The assessment result which only applied the diagnostic guide of the blood pressure measurement value and the assessment result which considered each risk factor were compared in order to confirm the effectiveness of the suggested model. The acquisition method of the assessment result involved modeling virtual IOT applications from the Linux system and conducting the experiment where suggested protocol message are sent a 100 times. In the experiment, a total of 1000 virtual sample patient data were produced per session and Figure 6 is a display of the result.

In Figure 6, (a) displays how the number of experiments where blood pressure was assessed as 'normal' decreased significantly (Normal+) when comprehensive consideration was given to risk factors. However, in case of 'warning' in (b), the number of assessment actually increased significantly (Warning+).
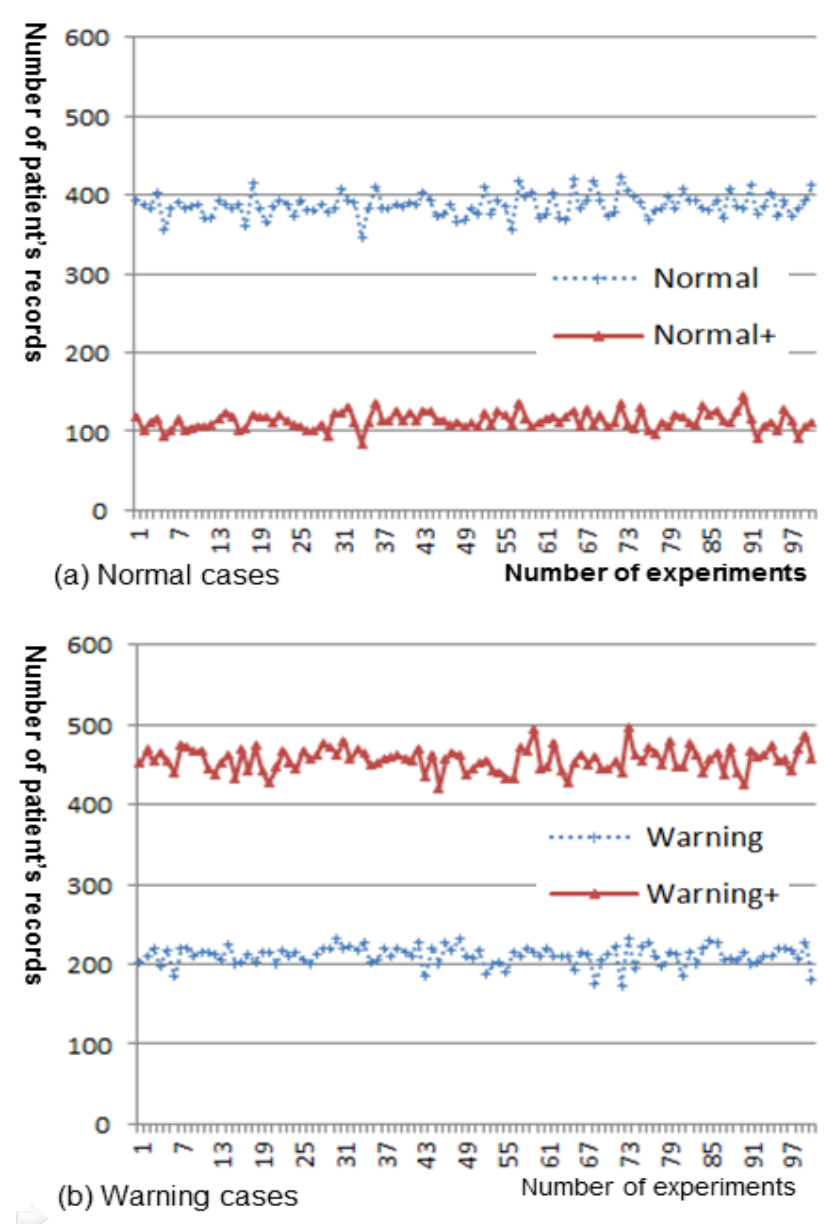


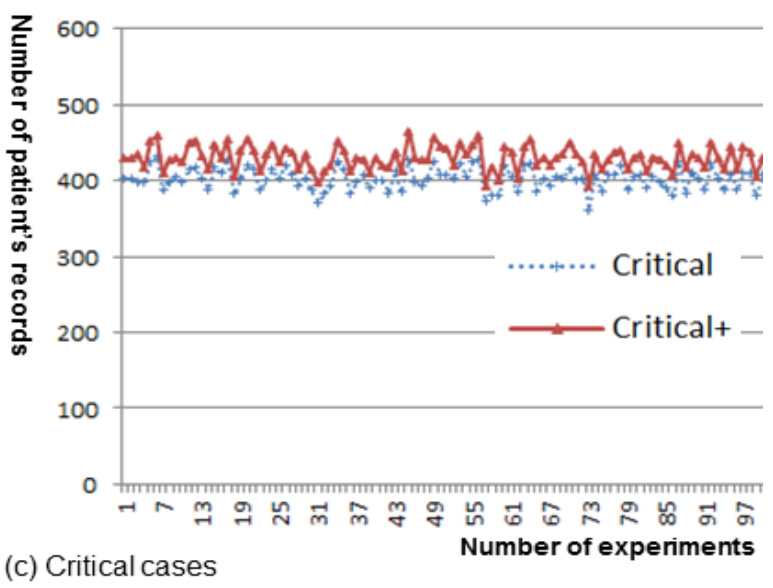

Figure 6. Evaluation of the experiments

This signifies how comprehensive assessment cause cases assessed as 'normal' to become assessed as 'warning,' In other words, even in cases when 'normal' results if assessed with only the measurement value, it results as 'warning' when assessed along with risk factors. In case of (c), minor difference is displayed. Assessment result of similar inclination resulted for glucose and obesity.

\section{Conclusion}

In this paper, proposed intelligent health service is enabled to overall assessment by the collaboration to exchange the risk factors such as blood pressure, obesity, diabetes, between personal health devices

As it was also displayed in the experiment result, it was identified that comprehensive assessment management provide more clear information about the patient's state in comparison to simple assessment management and it was considered as an effective model applied for patients to autonomously manage metabolic syndrome

\section{Acknowledgements}

This work was supported by the Gachon University research fund of 2013 (GCU-2013-R408).

\section{References}

[1] J. Morak, H. Kumpusch, D. Hayn, R. Modre-Osprian and G. Schreier, "Design and Evaluation of a Telemonitoring Concept Based on NFC-Enabled Mobile Phones and Sensor Devices", IEEE Transactions on Information Technology in Biomedicine, vol. 16, no. 1, (2012), pp. 17-23.

[2] V. Upkar, "Pervasive Healthcare and Wireless Health Monitoring. Mobile Network Application", KSII Trans. on Internet and Information Systems, vol. 12, (2007), pp. 113-127.

[3] J. P. C. R. Joel, R. E. P. Orlando and A. C. S. N. Paulo, "Biofeedback data visualization for body sensor networks", J. Network and Computer Applications, (2011), pp. 151-158.

[4] B. M. Lee and J. Ouyang, "Application Protocol adapted to Health Awareness for Smart Healthcare Service", In the proc. of International Workshop of Multimedia 2013, Advanced Science and Technology Letters, vol. 43, (2013), pp. 101-104.

[5] C. Min, W. Jiafu and L. Fang, "Machine-to-Machine Communications: Architectures, Standards and applications", KSII Trans. on Internet and Information Systems, (2012), pp. 480-497. 
[6] A. Jian, G. Xiao-lin, Y. Jian-wei, Z. Wen-dong and J. Jin-hua, "Research on a Mobile-aware Service Model in the Internet of Things", KSII Transaction on Internet and Information Systems, vol. 7, no. 5, (2013), pp. 1146-1165.

[7] Joint National Committee, "Prevention, Detection, Evaluation, and Treatment of High Blood Pressure", The $7^{\text {th }}$ Report of the JNC, U.S. Dept. of Health and Human Services, (2004).

[8] K. Richard, F. Ele, B. John and S. Michael, "The Metabolic Syndrome Time: for a Critical Appraisal. Diabetes Care", vol. 28, no. 9, (2005), pp. 2289-2304.

[9] American Diabetes Association, "Standards of medical care in diabetes. Diabetes Care 31 (Suppl 1)", (2008), pp. 12-54.

[10] "Definition and Diagnosis of Diabetes Mellitus and Intermediate Hyperglycemia", World Health Organization, (2006).

[11] M. L. James, C. Y. Donald, A. R. Joseph and M. D. Ronald, "Obesity: Assessment and Management in Primary Care”, American Family Physician, vol. 63, no. 11, (2001), pp. 2185-2196.

[12] A. Sarita and L. D. Manik, "Internet of Things - A Paradigm Shift of Future Internet Applications", Institute of Technology, Nirma University, (2011), pp. 1-7.

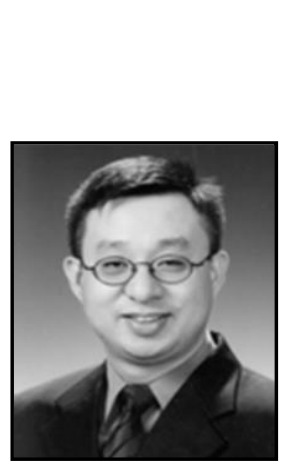

\section{Authors}

\section{Byung Mun Lee}

He received a B.S. degree in 1988 from Dongguk University, Seoul, Korea and a M.S. degree from Sogang University and a Ph.D. degree from University of Incheon Korea, in 1990 and 2007. He had worked for LG Electronics for 7 years. He is currently a professor in the department of Computer Science, Gachon University, South Korea as well as visiting scholar at California State University Sacramento, USA. His research interests are pervasive healthcare, its network protocol, IoT for healthcare, wireless sensor networks, operating system, etc.

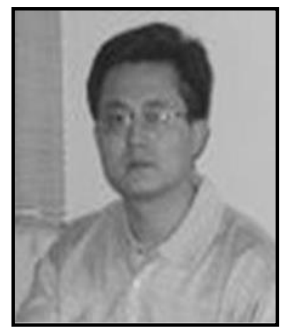

\section{Jinsong Ouyang}

$\mathrm{He}$ is Professor of Computer Science and Engineering at California State University Sacramento. He has over 20 years of experience in researching in the area of Distributed Systems. Prior to joining California State University Sacramento, USA. He was a researcher at Hewlett Packard Laboratory and a technology liaison between HP Labs and HP OpenView. He received his Doctor of Philosophy in Computer Science in 1997 from the University of New South Wales, Sydney, Australia. 